\title{
Dynamic Modeling and Simulation of Thermal Vacuum Test Platform for Spacecrafts Docking
}

\author{
Yanhe Zhu* \\ *Corresponding author \\ State Key Laboratory of Robotics and System \\ Harbin Institute of Technology \\ Harbin, China \\ e-mail: yhzhu@hit.edu.cn \\ Yu Zhang \\ State Key Laboratory of Robotics and System \\ Harbin Institute of Technology \\ Harbin, China \\ e-mail: zy_hitsz@163.com
}

\author{
Jie Zhao \\ State Key Laboratory of Robotics and System \\ Harbin Institute of Technology \\ Harbin, China \\ e-mail: jzhao@hit.edu.cn
}

\begin{abstract}
Spacecraft docking technology play an important role in the aerospace technology. Due to the spacecraft with variable attitude, so the docking process is complex and variable. Therefore, a comprehensive and systematic study of the spacecraft docking process is very necessary. This paper proposed the equivalent-mass principle and the test platform of spacecraft docking is established based on the equivalent-mass principle through the analysis of environmental characteristics of thermal vacuum. Furthermore, dynamic simulation on the test platform and the results data of simulation is obtained. These studies will facilitate the understanding of the spacecraft docking process and provide solid theoretical foundation for further study.
\end{abstract}

Keywords- spacecraft docking; thermal vacuum; buffering characteristic; dynamic modeling; dynamic simulation

\section{INTRODUCTION}

With the development of space technology, the spacecraft docking technology has become a key technique in construction of a space station. A lot of high-risk and accident exist in spacecraft docking process, simulation and experiment of docking mechanism is crucial. That also in order to ensure high success rate and reliability in spacecraft docking process[1-6]. Especially, when the space mechanism is used under thermal vacuum environment, it can lead the function failure of satellite or spacecraft. Such as that case was reported many times by news media[7-8]. The thermal vacuum of space has some peculiar characteristics that are high vacuum, high temperature and alternatively change of temperature. Thus, the emulated device comprise of thermal vacuum device, heat sink, vacuum air extractor and cooler[9-11]. Due to the limited volume of the thermal vacuum chamber, it brings more challenges for the design of thermal vacuum test platform of docking units.

The simulation of spacecraft docking process can be divided into three types - physical simulation, semiphysical simulation and digital simulation[12-16]. However, a physical prototype of the docking mechanism will cost too much. Furthermore the physical prototype will be damaged which is irreversible, if we use physical prototype to simulate the docking process under the condition of immature theory. Thus virtual prototype is widely used to simulate the process of spacecrafts docking, and greatly reduces the cost and danger of simulation process.

Virtual prototype is a novel system, new design method and fresh way, which can be reproduced the process of simulation system at some level. Thus, virtual prototype has very high similarity and authenticity when it was used to simulate the physical prototype[17-18]. By summarizing the current technology of virtual prototype and the conditions of the laboratory, we establish the equivalent-mass principle and built a test platform for spacecrafts docking based on the equivalent-mass principle. Furthermore, we use this model to simulate the process of docking of two spacecrafts through ADAMS software.

\section{EQUIVALENT-MASS PRINCIPLE OF SPACECRAFTS DOCKING}

\section{A. Physical description of the process of spacecratfs docking}

To perform the docking test of docking process of two spacecrafts (shown in Fig .1) with the docking velocities and the center connection of mass have the same orientation. Let us assume that the mass of two spacecrafts are $m_{1}$ and $m_{2}$ respectively. The velocities of pre-docking are denoted as $\mathrm{v}_{1}$ and $\mathrm{v}_{2}$.

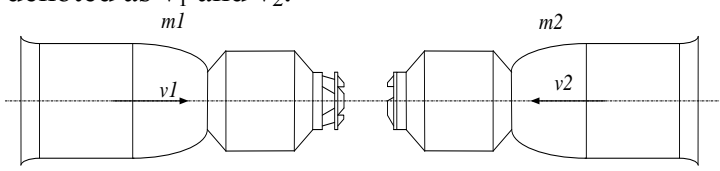

Figure 1. Docking process of two spacecrafts.

The theorem of momentum of docking process can be expressed as in (1).

$$
m_{1} v_{1}+m_{2} v_{2}=\left(m_{1}+m_{2}\right) v
$$

$v$ can be concluded as follows 


$$
v=\frac{m_{1} v_{1}+m_{2} v_{2}}{m_{1}+m_{2}}
$$

Where, $v$ is the post-docking velocity of spacecraft system.

The pre-docking and post-docking kinetic energy of two spacecrafts are shown as follows:

$$
\begin{aligned}
& A_{q}=\frac{1}{2}\left(m_{1} v_{1}^{2}+m_{2} v_{2}^{2}\right) \\
& A_{h}=\frac{1}{2}\left(m_{1}+m_{2}\right) v^{2}
\end{aligned}
$$

Substituting (2) to (4), we can get the equation as follows:

$$
A_{h}=\frac{\left(m_{1} v_{1}+m_{2} v_{2}\right)^{2}}{2\left(m_{1}+m_{2}\right)}
$$

Therefore the kinetic energy dissipated in docking process can be derived as in (6).

$$
A=A_{h}-A_{q}=\frac{m_{1} m_{2}\left(v_{1}-v_{2}\right)^{2}}{2\left(m_{1}+m_{2}\right)}
$$

\section{B. Equivalent-mass principle of docking process}

If the test platform has the same size as volume of two spacecrafts, there is no way to put the large volume test platform into the thermal vacuum chamber. The reasons are the volume of system of spacecrafts is lager and the bulk of thermal vacuum chamber is limited. Therefore, the volume of docking system can be greatly reduced when the test platform is designed based on the equivalent-mass principle. The equivalent model of space docking system, which based on the equivalent-mass principle, is shown as Fig .2.

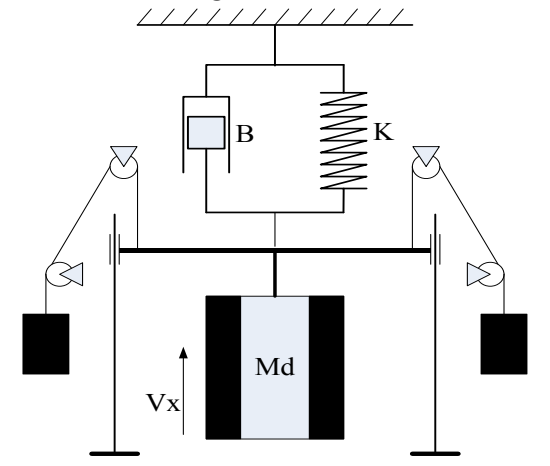

Figure 2. The equivalent model of docking process. (7)

We set, and then equation (6) can be simplified as in

$$
A=\frac{1}{2} m_{d} v_{d}
$$

Where, $m_{\mathrm{d}}$ and $v_{\mathrm{d}}$ are presented the mass and the velocity of the equivalent model respectively. $\mathrm{A}$ is the kinetic energy of the equivalent model.

\section{DYNAMIC SIMULATION BY SOFTWARE ADAMS}

\section{A. Dynamic modeling of docking process of test platform}

In this part, we used two methods to simulation of two spacecrafts docking. One of methods that the dynamic modeling with tow moving components, The other method is built a dynamic modeling with only one moving component.

\section{1) The spaceship collision model}

The spaceship is built based on the authentic docking process of two spacecrafts, each spacecraft has velocity. The schematic diagram is shown as Fig .1. As is shown in the Fig .3, which is the spaceship collision model with ADAMS.

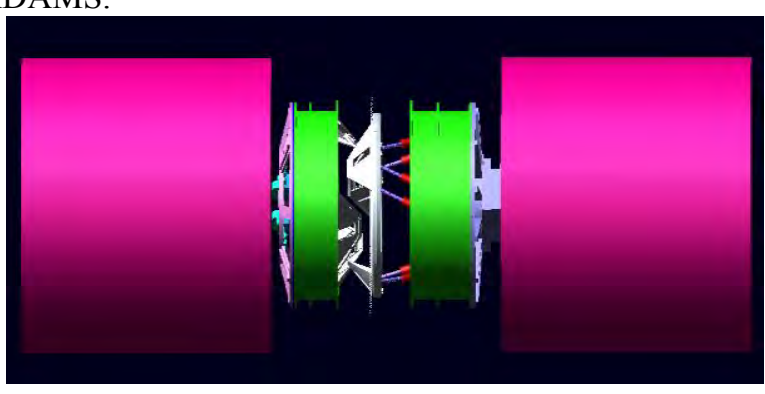

Figure 3. The spaceship collision model

\section{2) The direct equivalent model}

The direct equivalent model flow chart is shown as Fig .4. The 3-D geometry model of the test platform is shown as Fig .5, which is built based on the equivalentmass principle. Through the MECHANISM/Pro interface model of ADAMS to build the dynamic interactive environment of docking process, give the physical parameters of 3-D model, add constraints of joints. That will be needed for the next step. The dynamic interactive environment of ADAMS is shown as Fig .6.

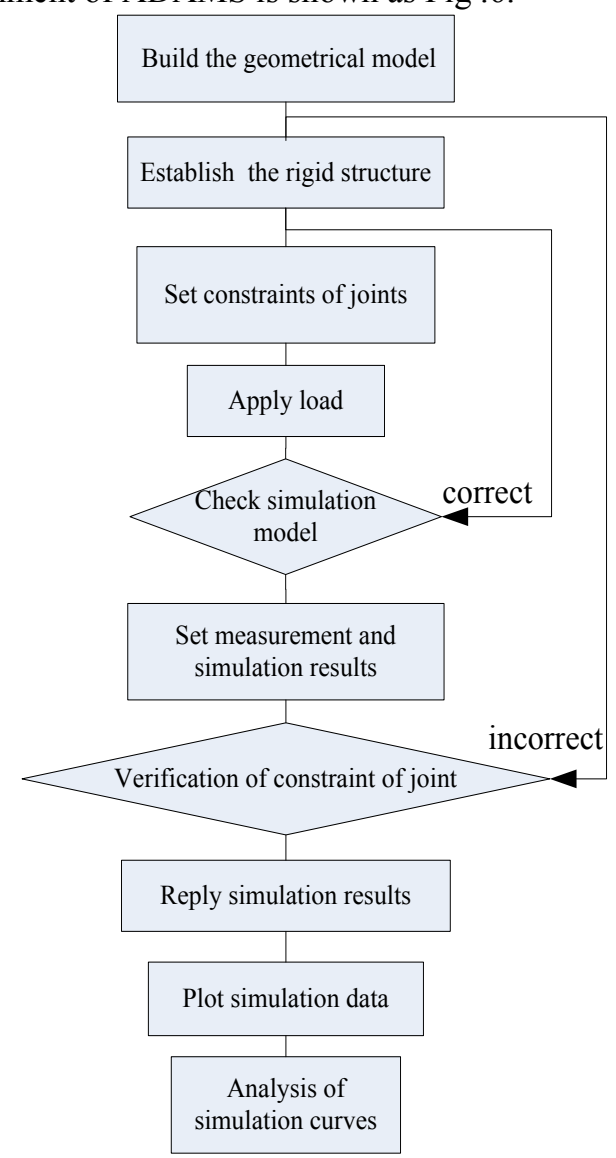

Figure 4. The direct equivalent model flow chart. 


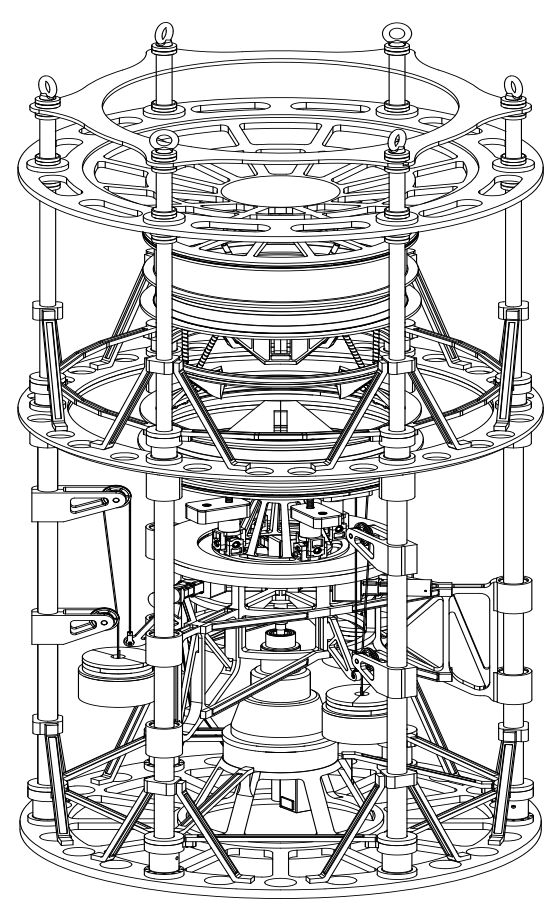

Figure 5. The 3-D geometry model of the test platform.

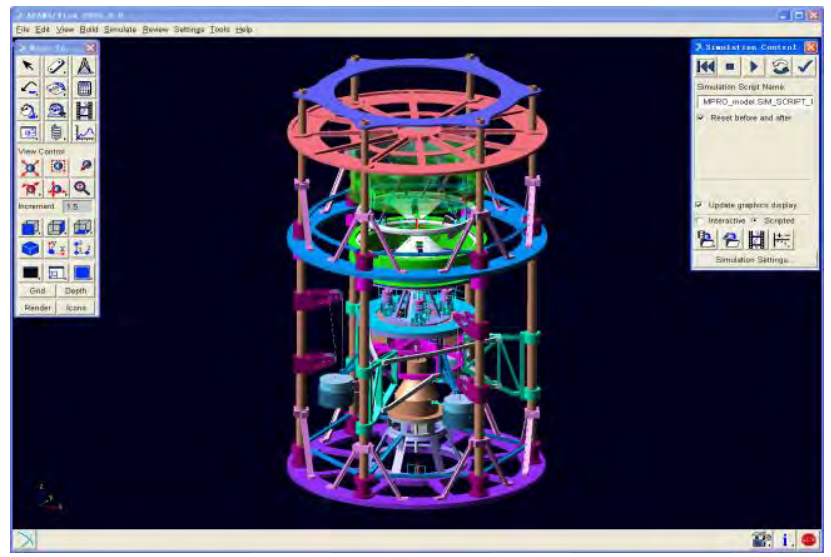

Figure 6. The dynamic interactive environment of ADAMS.

\section{B. Define connection of docking ring and buffering power of model}

The dynamic modeling of the docking ring of test platform is shown as Fig.7.
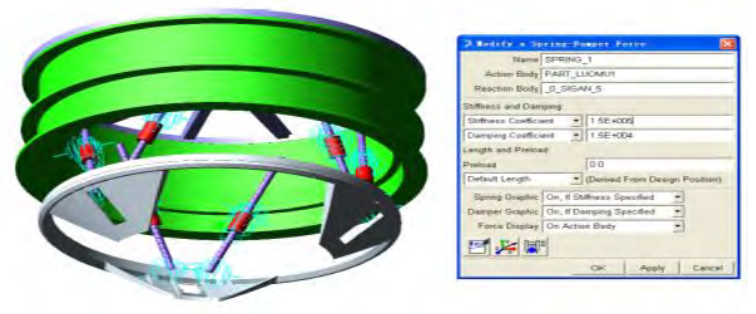

Figure 7. The dynamic modeling of docking ring.

Define the same spring and damping drive for nut of each screw, the parameters of spring and damping drive are shown as TABLE I. The origin of docking ring is located on the position of screw, which on the standard height.
Software ADAMS provides some graphic skits, which are Para solid and RAPID, for complex model. In this paper, we use EZAPID to detect the collision of spacecrafts docking, and select three hundred polygons to approximate the docking ring. Parameters of contact detection of docking ring are shown as Fig .8.

TABLE I. PARAMETERS OF SPRING AND DAMPING DRIVE
\begin{tabular}{|c|c|}
\hline Item & Value \\
\hline stiffness_mode & $($ linear) \\
\hline stiffness_coefficient & $(1.5 \mathrm{E}+004$ (DV_K)) \\
\hline damping_mode & (linear) \\
\hline damping_coefficient & $(2000.0$ (DV_B)) \\
\hline damping_spline & (no value) \\
\hline free_length_mode & (Design_Length) \\
\hline free_length & $(1.0)$ \\
\hline preload & $(0.0)$ \\
\hline
\end{tabular}

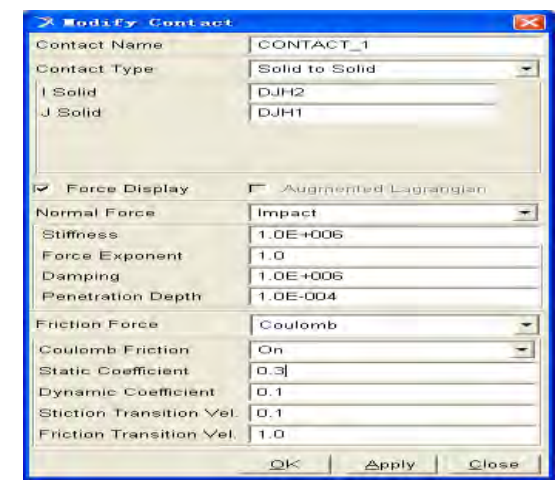

Figure 8. Parameters of contact detection of docking ring

\section{Dynamic simulation of docking process}

Define two models have the same conditions of simulation. They all also have the same initial conditions that are output measurement, initial state of model, stiffness of equivalent buffering model and parameters of damper. The model is simulated under two kinds of initial conditions, which are shown as TABLE II.

TABLE II. THE INITIAL CONDTIONS OF SIMULATION

\begin{tabular}{|c|c|c|}
\hline \multirow{2}{*}{ Number } & \multicolumn{2}{|c|}{ Parameters } \\
\cline { 2 - 3 } & Relative velocity & Relative attitude \\
\hline 1 & $400 \mathrm{~mm} / \mathrm{s}$ & 0 \\
\hline 2 & $400 \mathrm{~mm} / \mathrm{s}$ & $10^{\circ}(\mathrm{Pitch})$ \\
\hline
\end{tabular}

The comparisons of docking force is obtained from the simulation with ADAMS, they are shown as Fig .9 and Fig .10. 


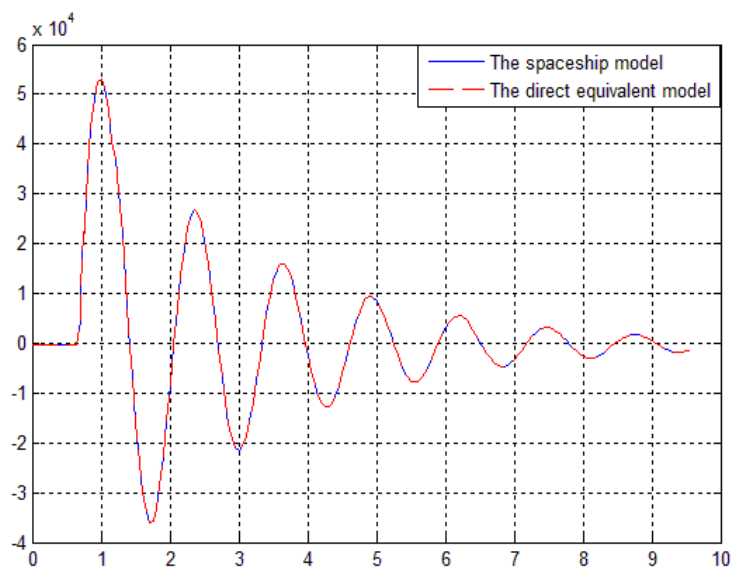

Figure 9. The comparison force with the first initial condition in TABLE II.

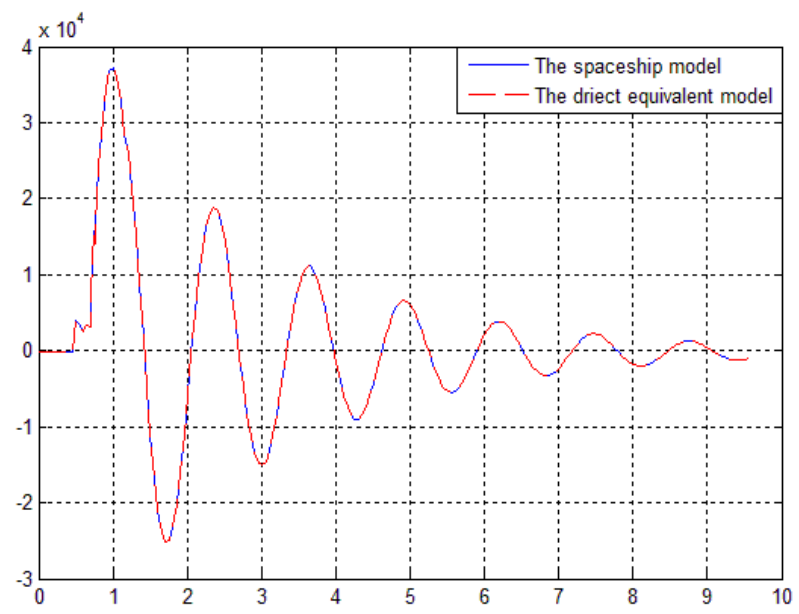

Figure 10. The comparisons force with the second initial condition in table II

Fig .9 and Fig .10 illustrate simulation forces of the process of docking, which under the two different kinds of initial conditions. The figure suggested that the fluctuation trend and periodic of oscillation. The amplitude of comparison with no fixed value, and the value moves around zero up and down. Due to space constraints, we only describe results of simulation conditions are $V x=0.4 \mathrm{~m} / \mathrm{s}$ and pitch $=10^{\circ}$. The comparison force has the same peaks and valleys. Amplitude of forces are both decreasing as the time and tending to zero eventually. The maximum amplitude values of force is $3.75 \times 10^{4} \mathrm{~N}$.

\section{CONCLUSION}

In this paper, we presented the equivalent-mass principle, which can be performed the docking process of two spacecrafts, with only one moving component. This method can greatly reduce the size of the test platform. Then we built the virtual prototype of test platform based on equivalent-mass principle and used this model to simulate the process of docking under different initial conditions with ADAMS. Furthermore, we obtained simulation results of docking forces. These studies will facilitate the understanding of the space docking process and provide solid theoretical foundation for further research.

\section{REFERENCES}

[1] ZOU Hua-wu, XU Feng, XIAO Yu-zhi. Control and Precision Appraisal of Dynamic Test Platform for Space Docking.[J]. Jurnal of Manned Space. vol.20, no.1, pp. 50-57, 2014.

[2] TIAN Hao, ZHAO Yang, ZHANG Da-wei. Modeling and Analysis of Motion Simulator of Comprehensive Experiment Platform in Space Docking Mechanism[J]. Acta Astronautica, vol.28, no.4, pp. 99-1001, 2007.

[3] XU Zhi-gang, XIN Li-ming, ZHAO Ming-yang. Gravity Balance Technique of Active Docking Ring for Space Docking Test Table[J]. Chinese Jounal of Scientific Instrument. 2009, vol.30, no.6, pp: 1140-1144.

[4] XIAO Zhi-yu, ZOU Hua-wu, XU Feng. Reasearch on The Dynamic Modeling and Simulation of Docking Test Platform.[J] 2010, vol.31, no.3, pp: 674-679.

[5] FENG Xue, TANG Qian-gang, WANG Li-zai. Test Principles of Semi-physical Simulation of Test Platform with 6-DOF[J]. Jurnal of Manned Space. 2007, no.1, pp: 24-28.

[6] ZHANG Guang-yu, ZHANG Chong-jun, ZHAO Xue-zeng. Gravity Balance Technique Installed on Six-freedom Spacecraft Docking Test Table[J]. Journal of Beijing University of Aeronautics and Astronautics. 2004, 30(12):1186-1190.

[7] ECSS-E-10-03A-2002. Space engineer[S].

[8] YANG Hui, BAI Hua, LIU Yan-fang, HA Wen-hui. Evaluation Method of Components for Thermal Vacuum Test of Space[J], Electronics and Packing, vol.11, no.6, pp. 31-35, 2011.

[9] JI Xin-yan, LIU Guo-qing, PEI Yi-fei. The Current State of Effectiveness Research on Temperature Environment and Thermal Vacuum Test, Vacuum and Cryogenics, pp. 600-604, 2011.

[10] ZHANG Lei, LIU Wei-cheng, WEI Yong-qiang, Debugging Summaries of Emulated Device for Thermal Vacuum Environment[J], GanSu Science and Technology. vol.27, no.19, pp. 100-102, 2011.

[11] LIU Feng. Control Method of Thermal Vacuum Test[J]. Spaceflight Environment Engineering, vol.20, no.3, pp. 1-6, 2003.

[12] LIN Lai-xing. The Rendezvous and Docking Technology of Space[M]. National Defence of Industry Press, Beijing, 1995.

[13] YuWei Yang, Lei Qu Guangji. Dynamics Analysis and Simulation of Spacecraft Docking Mechanism[J]. Journal of Dynamics and Control, vol.2, no.2, pp.38-42, 2004.

[14] LAI Yi-nan, ZHAO Yang, TIAN Hao. Dynamic Characteristics Simulation for Spacecraft Docking Five-Freedom Test Table[J] Acta Simulata Systematica Sinica, 2005, vol17, no.11, pp.25732576 .

[15] XueXiao Wang, ZhangQiong Chen, QingRui Zhu. Docking Dynamics of a Spacecraft[J]. Journal of Astronautics,

[16] YingZi Guan, NaiGang Cui, YuHua Liu. Numerical Simulation of Spacecraft Docking Dynamics[J], Jouran of Harbin Institute of technology, vol.31, no.4, pp.121-124, 1999.

[17] TongLi Chang, DaCheng Cong, ZhengMao Ye, JunWei Han. Research on simulation of semi-physical simulation system on the ground for space docking, Acta Aeronautica. vol.28, no.4, pp. 975 980, 2007.

[18] N.Zuech. Understanding and Applying Machine Vision[M]. New York, Marcel Dekker, 2000

[19] HUANG Hai-bing, LI Hai-yang, WANG Hua, TANG Guo-jin. Modeling Research of Influence on Docking Combined Spacecraft's Initial Attitude[J]. Journal of National University of Defense Technology. 2010, vol.32, no.3, pp: 84-88.

[20] GUANG Yi-zi, KANG Wei-min, CUI Nai-gang. Simulation of Initial Capture Period during Docking Process[J]. Acta Simulata Systematica Sinica. 2000, vol.12, no.6, pp:664-667.

[21] DU Ya-juan.Scheme of Control System in Half-physical Simulation Comprehensive Test-bed[J].Computer Engineering. 2010, vol.36, no.22, pp:233-235. 